\title{
A Proactive Mechanism for Selective Suppression of Response Tendencies
}

\author{
Weidong Cai, Caitlin L. Oldenkamp, and Adam R. Aron \\ Department of Psychology, University of California, San Diego, La Jolla, California 92093
}

While most research on stopping action examines how an initiated response is stopped when a signal occurs (i.e., reactively), everyday life also calls for a mechanism to prepare to stop a particular response tendency (i.e., proactively and selectively). We hypothesized that human subjects can prepare to stop a particular response by proactively suppressing that response representation in the brain. We tested this by using single-pulse transcranial magnetic stimulation and concurrent electromyography. This allowed us to interrogate the corticomotor excitability of specific response representations even before action ensued. We found that the motor evoked potential of the effector that might need to be stopped in the future was significantly reduced compared with when that effector was at rest. Further, this neural index of proactive and selective suppression predicted the subsequent selectivity with which the behavioral response was stopped. These results go further than earlier reports of reduced motor excitability when responses are stopped. They show that the control can be applied in advance (proactively) and also targeted at a particular response channel (selectively). This provides novel evidence for an active mechanism of suppression in the brain that is setup according to the subject's goals and even before action ensues.

\section{Introduction}

The ability to stop a response is important in everyday life. Much research has investigated this using experimental paradigms, such as go/no-go and stop-signal tasks (Verbruggen and Logan, 2009). However, these paradigms are limited by the fact that stopping is externally signaled (reactive) and also may have general effects over the motor system (global) (Hoshiyama et al., 1997; Sohn et al., 2002; Coxon et al., 2006, 2007; Badry et al., 2009). Yet, real life also requires one to stop a specific response (i.e., selectively). This can be examined with the cued selective stopping task (Fig. 1a) (Aron and Verbruggen, 2008; Claffey et al., 2010). Each trial begins with a cue such as "Maybe Stop Right" (MSR) or "Maybe Stop Left" (MSL), instructing the subject to prepare to stop a particular hand. This is followed by a go signal, requiring simultaneous bimanual responses. Occasionally, a subsequent stop signal occurs, requiring the subject to stop one response and to continue with the other. Behaviorally, the selectivity of stopping can be measured by the size of the delay of the continuing hand on a stop trial relative to moving that hand on a no signal (go) trial. A key enabler of such behaviorally selective stopping is that one has a goal in mind of which response(s) to stop, i.e., proactive control.

Here we consider two possible models underlying proactive control. The suppression model assumes there is proactive suppression of the response representation that might need to be

Received Dec. 2, 2010; revised Feb. 14, 2011; accepted Feb. $28,2011$.

Author contributions: W.C. and A.R.A. designed research; W.C. and C.L.O. performed research; W.C. and C.L.O. analyzed data; W.C., C.L.O., and A.R.A. wrote the paper.

Funding was gratefully received from the Alfred P. Sloan Foundation, National Institutes of Health-National Institute on Drug Abuse Grant DA026452, and National Science Foundation Grant 0921168.

Correspondence should be addressed to Adam R. Aron, Department of Psychology, University of California San Diego, 9500 Gilman Drive, La Jolla, CA 92093. E-mail: adamaron@ucsd.edu.

DOI:10.1523/JNEUROSCI.6292-10.2011

Copyright $\odot 2011$ the authors $\quad 0270-6474 / 11 / 315965-05 \$ 15.00 / 0$ stopped. The facilitation model assumes there is proactive facilitation of the response representation that will not need to be stopped. The difference between these models relates to an important debate in cognitive control research. Is stopping action achieved via active suppression or is it achieved via withdrawal of facilitation or facilitation of alternatives? (MacLeod et al., 2003; Nieuwenhuis and Yeung, 2005; Aron, 2007; Cepeda and Munakata, 2007; Curtis and D'Esposito, 2008). Consistent with the suppression model, studies with transcranial magnetic stimulation (TMS) have shown reduced corticomotor excitability when a response is stopped (Hoshiyama et al., 1997; Sohn et al., 2002; Coxon et al., 2006; Claffey et al., 2010). However apart from the study by Claffey et al. (2010) those studies examined reactive rather than proactive control. Thus it is possible that the reduced excitability related to a withdrawal of facilitation rather than active suppression. The study by Claffey et al. (2010) examined proactive control but it did not include an appropriate baseline to distinguish between suppression and facilitation models.

Here we used TMS to examine proactive control and to compare suppression and facilitation models. On each trial, shortly following the cue, we delivered a TMS stimulus to the left primary motor cortex and recorded the motor evoked potential (MEP) from the right hand (Fig. 1b). To verify the suppression model, we included a "Null" cue baseline condition, which required no response (cf. Hoshiyama et al., 1997).

The suppression and facilitation models make different predictions about corticomotor excitability immediately following the cues. If there is suppression then, when the cue is MSR, the MEP for the right hand should be reduced compared with null (Fig. 1c). In contrast, if there is facilitation then, when the cue is MSR, the MEP for the right hand should not be different compared with null; instead when the cue is MSL the MEP for the right hand should be increased compared with null (Fig. 1d). 


\section{Materials and Methods}

Subjects. Sixteen subjects (10 female, all right handed, 18-24 years) provided consent at the University of California at San Diego and passed TMS safety screening.

Apparatus, task, and procedure. Subjects sat $50 \mathrm{~cm}$ in front of a 19-inch monitor with hands on a four-keypad response device (2 vertical and 2 horizontal keypads). Each trial began with a cue, "Maybe Stop Left" (MSL), "Maybe Stop Right" (MSR) "No Stop" or "Null” with equal likelihood, written in white text on a black background. The MSL or MSR cues instructed subjects to prepare to stop the left or right hand, respectively. The no stop cue instructed subjects to prepare to respond to the go signal (no stop signals presented). The null cue instructed subjects to rest. The cue was presented for $500 \mathrm{~ms}$, after which the screen turned black for $1.5 \mathrm{~s}$. On trials with MSL, MSR or no stop cues, four circles were presented in a horizontal row, each $2.3^{\circ}$ visual angle in diameter. The two inner circles were separated by $4.6^{\circ}$ and each inner was separated from the outer ones by $1.2^{\circ}$. The four circles corresponded to left little and index and right index and little fingers. On go trials, either two inner or outer circles were filled blue (go signal), while the others were filled white. Subjects made quick bimanual responses simultaneously to the two blue circles within a $1 \mathrm{~s}$ response window using the corresponding fingers. The index fingers were moved inward to press vertical keys (optimal for EMG) and little fingers were pressed downward. To prevent subjects from delaying the cued hand, the text "decoupled" was presented after responses for which the difference in reaction time (RT) for the two hands was $>70 \mathrm{~ms}$.

Occasionally (33\%), a red "X" (stop signal) was presented shortly after the go signal between the two inner circles (probe trials). The delay between the go and stop signal is called the stop-signal delay (SSD). The SSD was adjusted dynamically: decreasing by $50 \mathrm{~ms}$ for a failed stop and increasing by $50 \mathrm{~ms}$ for a successful stop. When a stop signal occurred, subjects tried to withhold the response on the cued hand (e.g., right hand for "Maybe Stop Right") and to continue the response on the non-cued hand (e.g., left hand for "Maybe Stop Right").

There were 6 blocks of 80 trials. Each block contained 20 trials for MSL, MSR, no stop, and null cues. Cues were randomly intermixed within each block. Go trials and probe trials were pseudo-randomly mixed and there were no more than 2 consecutive probe trials. A singlepulse TMS stimulus was delivered at either 800,500 , or $200 \mathrm{~ms}$ before the go signal, or in the intertrial interval (ITI) (250 ms before the cue). In a total of 480 trials, there were 48 TMS stimuli in the ITI (equiprobably for each of four conditions). If there was a TMS stimulus in the ITI, then there was none after the cue for the trial. Overall, for each of the four conditions, there were 108 TMS stimuli after the cue for the trial. For the MSL and MSR conditions, 72 stimuli occurred on go trials and 36 on probe trials. The ITI varied from 2 to $3 \mathrm{~s}$. We realized that the no stop condition is effectively redundant in addressing the suppression versus facilitation models, so it is dropped from the analysis.

$E M G$ recordings. Surface EMGs were recorded from the first dorsal interosseous of the right hand using a method and equipment identical to those described by Claffey et al. (2010).

TMS. TMS was delivered using a MagStim 200-2 system (MagStim) and a $70 \mathrm{~mm}$ figure-of-eight coil. The coil was placed $5 \mathrm{~cm}$ lateral and 2 $\mathrm{cm}$ anterior to the vertex and repositioned while delivering a TMS stimulus to locate the position where the largest MEPs were observed consistently. Next, the maximum MEP size was determined by increasing stimulus intensity in 3\% increments until the MEP amplitude no longer suppression and facilitation models.
TMS stimulus delivered at 800 , Response Response TMS stimulus delivered
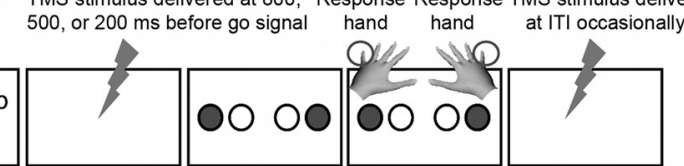

Continuing Stopped

hand hand

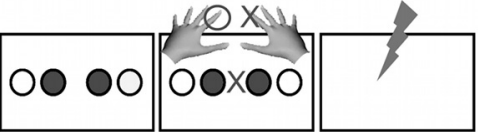

TMS stimulus delivered at same

times as in Go and Probe trials

Probe trials
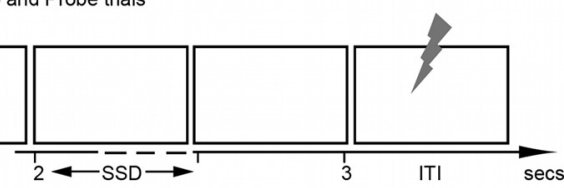

acilitation

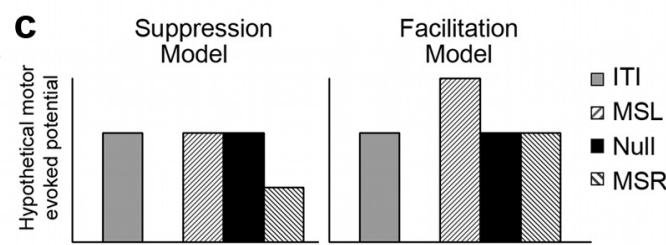

$M S L$

- Null

Figure 1. $\quad \boldsymbol{a}$, The selective stopping paradigm. Trials are shown for MSR and null conditions. A cue was followed by a delay and - a row of four circles. If the two outer circles were blue, two responses were simultaneously initiated with little ( was delivered over left primary motor cortex. MEPs were recorded from the right hand. $c$, Hypothetical MEP results for the

increased. Finally, the TMS stimulus intensity was adjusted to produce an MEP that was approximately half of the maximum MEP amplitude while the subject was performing the task in a practice session. This was the intensity used during the experiment proper ( $54 \pm 10 \%$ maximum stimulator output on average, which was $\sim 112 \%$ of resting motor threshold: $48 \pm 9 \%)$.

Behavioral analysis. For "Maybe Stop Left" and "Maybe Stop Right" cues separately, we calculated several indices (Table 1) (Aron and Verbruggen, 2008): go cue RT, RT of the cued response on go trials; go alternative RT, RT of the alternative response on go trials; go cue lead, go cue RT minus go alternative RT; decouple rate, proportion of go trials where the difference between go cue RT and go alternative RT is $>70 \mathrm{~ms}$; probe accuracy, proportion of probe trials where subjects stopped the cued response and continued the other; probe fail rate, proportion of probe trials where subjects failed to stop the cued response; direction errors, proportion of probe trials with wrong response stopped; probe alternative RT, RT of the alternative response on correct probe trials; stopping interference effect, probe alternative RT minus go alternative RT; SSRT, stop-signal RT (go cue RT minus SSD).

TMS analysis. MEPs were identified from the EMG using custom Matlab software (MathWorks). MEP sweeps began $200 \mathrm{~ms}$ before the TMS stimulus. Trials were excluded if the root mean square EMG in the 100 ms before the TMS stimulus was $>10 \mu \mathrm{V}$. The top and bottom $10 \%$ of MEPs in each cue condition and the ITI were trimmed. Mean MEP amplitude was calculated for cue condition, ITI, and TMS stimulus time for each subject.

To examine whether the muscle of interest was "at rest" before TMS stimulation, we calculated root mean square EMG activity in the $100 \mathrm{~ms}$ before the TMS stimulus for each condition.

\section{Results}

\section{Behavior}

Averaging over both MSR and MSL conditions, go RT was 0.576 s, SSRT was $0.297 \mathrm{~s}$, and the stopping interference effect was 0.180 s. The subjects were clearly using the MSR and MSL cues effec- 
Table 1. Behavioral results

\begin{tabular}{lccl}
\hline & MSL & MSR & Significance $(p)$ \\
\hline Go cue RT (seconds) & $0.576(0.080)$ & $0.577(0.076)$ & 0.915 \\
Go alternative RT (seconds) & $0.577(0.079)$ & $0.572(0.080)$ & 0.271 \\
Go cue lead (seconds) & $0.001(0.007)$ & $-0.005(0.009)$ & 0.113 \\
Go decouple rate (\%) & $7.4(5.3)$ & $8.0(6.4)$ & 0.523 \\
Probe accuracy (\%) & $57.8(7.0)$ & $56.8(7.1)$ & 0.252 \\
Probe fail rate (\%) & $36.1(5.6)$ & $36.8(6.1)$ & 0.523 \\
Direction errors (\%) & $3.5(3.3)$ & $2.8(4.4)$ & 0.510 \\
Probe alternative RT (seconds) & $0.761(0.159)$ & $0.747(0.156)$ & 0.257 \\
Stopping interference (seconds) & $0.184(0.119)$ & $0.175(0.123)$ & 0.437 \\
SSD (seconds) & $0.282(0.058)$ & $0.278(0.061)$ & 0.513 \\
SSRT (seconds) & $0.294(0.040)$ & $0.299(0.036)$ & 0.539
\end{tabular}

Behavioral performance was highly similar regardless of whether the cue was MSL or MSR.
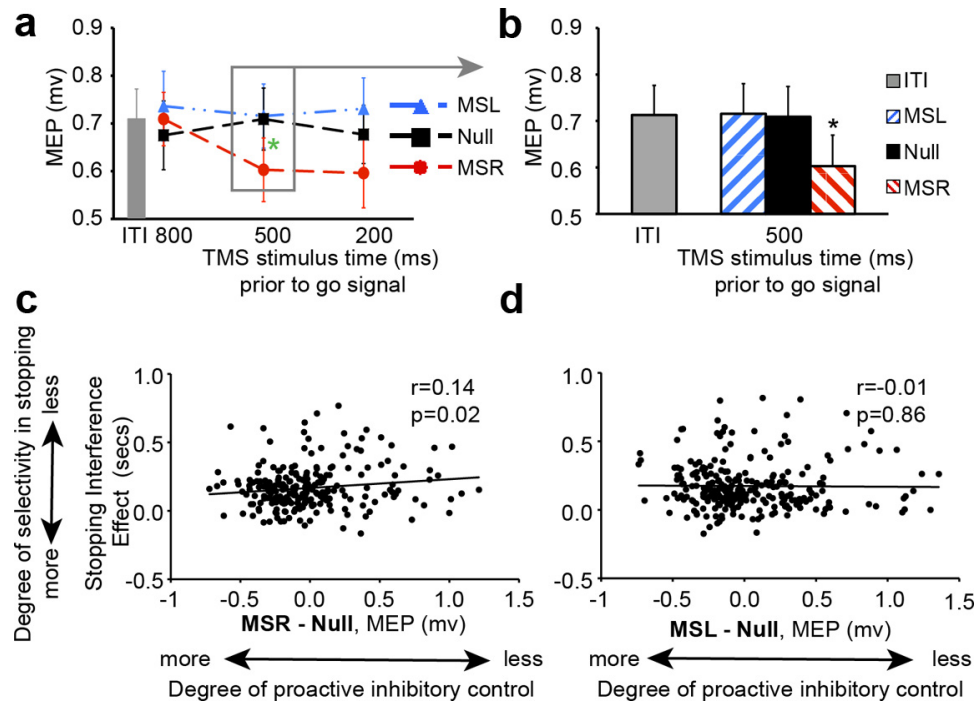

Figure 2. $\quad \boldsymbol{a}$, Preparing to stop the right hand (MSR) leads to suppression of right hand excitability beneath baseline (null) even before the response is initiated (Note: this analysis includes all trials regardless of whether they are subsequently go or stop.) $\boldsymbol{b}$, The MEP results match the suppression model in Figure 1c. MEP data are shown for the $500 \mathrm{~ms}$ time point alone - see dotted rectangle in $\boldsymbol{a}$. $c$, Relation between neural suppression and behavioral stopping. When preparing to stop the right hand, the MEP suppression of that hand (MSR minus null) correlates with the subsequent selectivity of stopping (thestopping interference effect). $\boldsymbol{d}$, When preparing to stop the left hand, the MEP suppression of the right hand (MSL minus null) does not correlate with the stopping interference. The observations in c and d reflectsuccessful stop trials only, and these are pooled across all subjects. The stopping interference effect is the probe alternative RT minus the mean of the go alternative RT for that subject. The neural measure is the size of the MEP on that trial minus the mean of the MEPs for null trials at the corresponding time point $(800,500$, and 200$)$ for that subject. Error bars show SD; ${ }^{*} p<0.01$.

tively as the number of trials on which they stopped the incorrect response was only $\sim 3.2 \%$. Importantly, behavioral values for the MSR and MSL conditions were highly similar, with no significant differences (Table 1). This allows a clear-cut comparison between the suppression and facilitation models using the MEP data. We note that there was no significant interaction between cue (MSL vs MSR) and hand (go cue vs go alternative), even when decoupled trials were included $(p>0.1)$.

\section{TMS}

The suppression model predicts smaller MEPs for MSR than null (Fig. 1c). The facilitation model does not-it instead predicts that MEPs for MSL are greater than null.

We tested the suppression model using ANOVA with two cues (MSR and null) and three time points (800, 500, and $200 \mathrm{~ms}$ before go signal). There was a significant interaction, $F_{(2,30)}=$ $6.11, p=0.006$ (Fig. $2 a$ ), and main effect of time point, $F_{(2,30)}=$ $3.65, p=0.038$, but no significant main effect of cue $(p=0.11)$ (Fig. 2a). Subsequent $t$ tests showed that MEPs for MSR were significantly smaller than null at $500 \mathrm{~ms}, t_{(15)}=3.73, p=0.002$ (significant after Bonferroni correction for three tests, i.e., $p<$ 0.0167 ), but not at $200 \mathrm{~ms}, t_{(15)}=2.06, p>0.0167$ (Fig. $2 b$ ), or at $800 \mathrm{~ms}, t<1$. Notably, there were no differences in pre-TMS uscle excitability. The EMG in the $100 \mathrm{~ms}$ before TMS showed ight hand was equally at rest for MSR and null conditions ( $p$.2). Importantly, the corticomotor excitability for null trinull effect is best characterized as suppression rather than differential facilitation compared with the ITI baseline.

tested the facilitation model using ANOVA with two cues (MSL and null) and three time points (800, 500, and $200 \mathrm{~ms}$ before go signal). There was no interaction of cue (MSL vs null) and time point and no main effects, all $p$ values $>0.40$ (Fig. $2 a$ ). Again, the righthand muscle was equally at rest for MSL and null conditions in the $100 \mathrm{~ms}$ before the TMS stimulus $(p>0.2)$.

These results clearly support the suppression model (Fig. 1c). When the subject is given a goal to prepare to stop the right hand, this is done by proactively suppressing the right-hand response representations.

If the proactive suppression is functionally important, it should relate to how well people target a particular tendency at the behavioral level (i.e., the selectivity of stopping). For probe trials, we correlated an MEP index of proactive suppression (MSR MEP minus mean null MEP) against a behavioral index of selective stopping, i.e., the stopping interference effect. Again, for probe trials, this is the RT on the continuing hand minus the mean RT of that hand on go trials for each subject (Fig. 1a). For trials on which there was more proactive suppression, stopping was more selective, $p=0.02$ (Fig. $2 c$ ). Moreover, this was specific to the hand that needed to be stopped. A similar analysis of MEPs from the right hand, but for the MSL condition, did not show a positive correlation, $p=0.86$ (Fig. $2 d$ ). The correlation was significantly stronger for MSR compared with MSL $(p<0.05)$. [Note: this analysis is based on pooled trials from all subjects because there were too few successful stop trials per subject. This restricts the statistical generalizability of this finding to the current sample.]

\section{Discussion}

On each MSL or MSR trial, subjects used a cue to prepare to stop a specific hand. TMS was delivered after the cue and MEPs were recorded from the right hand. There were two main findings. First, when the cue was MSR, then the right hand MEPs were reduced compared with the null condition, especially at the 500 ms time point. This clearly matches the prediction of the suppression model-subjects can engage a proactive and selective mechanism to begin to suppress particular response channels in the brain even before movement ensues. Second, the degree of proactive suppression (i.e., the MSR-null difference) predicted the subsequent selectivity of stopping at the behavioral level (albeit when pooling trials across subjects). On trials with greater pro- 
active neural suppression, the subjects stopped one hand and continued with the other with shorter delay. This shows that the neural suppression in the foreperiod is functionally important for subsequent behavior.

The results speak to an important debate about the status of an active mechanism of suppression or "inhibitory control." While some authors regard inhibitory control as a cardinal function of cognitive control (Diamond, 1990; see Smith, 1992, for historical background), others have expressed skepticism (MacLeod et al., 2003; Nieuwenhuis and Yeung, 2005; Cepeda and Munakata, 2007; Curtis and D'Esposito, 2008). For example, the skeptics argue that stopping can be achieved by the withdrawal of facilitation. While several TMS studies have demonstrated reduced MEPs for no-go or stop trials (Sohn et al., 2002; Coxon et al., 2006; Claffey et al., 2010) and even relative to a rest baseline (Hoshiyama et al., 1997), those studies examined reactive control, and so it is uncertain whether the underlying mechanism was active suppression or a withdrawal of facilitation. A study by Claffey et al. (2010) used a methodology similar to that used in the current study, finding reduced MEPs for MSR compared with MSL in the foreperiod, but did not include a null baseline and could not compare with the ITI because the trials were blocked. Here we clearly show that the MEP is reduced from the right hand when subjects prepare to stop the right hand, and this is relative to the null baseline as well as the ITI. This is strong evidence for active suppression.

The suppressive effect was temporally specific, occurring at $500 \mathrm{~ms}$ but not at $800 \mathrm{~ms}$ before the go signal. Since the delay between the cue and the go signal was fixed, the subjects likely estimated when the go signal would occur and initiated the proactive suppression at that point. Our findings also show specificity at the motor level. When the cue was MSR, there was suppression of the right hand, but this was not the case when the cue was MSL.

Importantly, the pattern of data when comparing MSR and MSL with the null condition argues against alternative explanations for the "suppression" on MSR trials in terms of temporal preparation (Davranche et al., 2007) or "impulse control" (Duque et al., 2010) in relation to the upcoming go signal. If the MEP reduction is merely a result of temporal preparation or impulse control, there should be an MEP reduction for the MSL, too, which we did not see. Instead, we argue that the suppression is the signature of proactive and selective suppression of the response representation that may need to be stopped in the future.

In this study, subjects were forced to use information in working memory to prepare to stop selectively. However, the stopping interference effect was large, at $180 \mathrm{~ms}$ on average. Nevertheless, we think subjects were mostly stopping in a truly selective manner, rather than stopping all output and then reinitiating the continuing hand. First, some subjects had values as small as $70 \mathrm{~ms}$. Second, the probe method inflates the estimates of the stopping interference effect. On trials where subjects fail to prepare to stop selectively, they presumably stop all response output and reinitiate the continuing response. Even a few such trials will substantially increase the stopping interference effect. Third, the true stopping interference effect is likely to be considerably less than the estimated value. This is because it is derived from the mean of the probe alternative RT minus the mean of the go alternative RT. Yet the probe alternative RT comes from a distribution of trials on which the other response was stopped, whereas the go alternative RT comes from the entire distribution. Fourth, we observed a significant correlation between the neural suppression effect (MSR-null) and the stopping interference effect. Thus, there are strong grounds to believe that many subjects were stopping selectively much of the time.

We speculate that the neural basis of proactive and selective suppression is a frontostriatal circuit, probably including the dorsolateral prefrontal cortex and the caudate nucleus. This links working memory to inhibitory control via the "indirect pathway" of the basal ganglia. The basis for this idea is as follows. First, working memory is evidently important for selective stopping (Aron and Verbruggen, 2008; Claffey et al., 2010) and relies on dorsolateral sectors (Müller and Knight, 2006; D’Esposito, 2007). Second, selective stopping is likely achieved via the indirect pathway of the basal ganglia including the striatum, as this has the requisite neural specificity to allow targeting of particular response tendencies (Mink, 1996). Third, the dorsolateral prefrontal cortex and striatum are connected via a classic "associative" loop (Alexander et al., 1990). Thus, we propose that subjects encode the stopping goal "Maybe Stop Right" into working memory, which then creates an influence over the relevant response channels in the basal ganglia (Fig. 3). This creates a proactive and selective inhibitory set that can be triggered when a stop signal occurs (Aron, 2010). However, there are other candidate neural mechanisms for selective suppression. For example, MEP suppression observed during the foreperiod of a reaction time task has been related to changes at the spinal level (Prut and Fetz, 1999; Duque et al., 2010). The present results cannot determine whether particular response channels are suppressed via the indirect pathway of the basal ganglia, or, for example, via direct descending projections to the spinal motor neuron pool from premotor cortex. Future research is needed to test our frontostriatal prediction, for example, by using functional magnetic resonance imaging to identify dorsolateral prefrontal cortex and basal ganglia activation and using disruptive TMS of the dorsolateral prefrontal cortex to demonstrate necessity. 
The current behavioral paradigm could serve as a more ecologically valid model for neuropsychiatric disorders. While much research has modeled failures of urge control in these disorders as problems with stopping a response reactively (Chambers et al., 2009), the control failures in such patients are probably more closely related to weaknesses in setting up the control system than in stopping a motor response per se. Here we show how working memory for stopping goals and the ability to stop the correct response are linked.

In summary, we have shown that when a subject uses a goal to prepare to stop a particular hand, the corticomotor excitability measured from that hand is reduced compared with baseline, even before action ensues. This excitability reduction is better explained by top-down active suppression of response channels rather than differential facilitation. Moreover, the degree of proactive suppression predicted the subsequent selectivity of behavioral stopping. These results speak to an important debate in cognitive control research about active mechanisms of suppression; they make a link between working memory and inhibitory control; and they motivate new behavioral and physiological paradigms for investigating the control of response tendencies in impulse control disorders.

\section{References}

Alexander GE, Crutcher MD, DeLong MR (1990) Basal gangliathalamocortical circuits: parallel substrates for motor, oculomotor, "prefrontal" and "limbic" functions. Prog Brain Res 85:119-146.

Aron AR (2007) The neural basis of inhibition in cognitive control. Neuroscientist 13:214-228.

Aron AR (2010) From reactive to proactive and selective control: developing a richer model for stopping inappropriate responses. Biol Psychiatry. Advance online publication. Retrieved February 14, 2011. doi:1016/j.biopsych.2010.07.024.

Aron AR, Verbruggen F (2008) Stop the presses: dissociating a selective from a global mechanism for stopping. Psychol Sci 19:1146-1153.

Badry R, Mima T, Aso T, Nakatsuka M, Abe M, Fathi D, Foly N, Nagiub H, Nagamine T, Fukuyama H (2009) Suppression of human corticomotoneuronal excitability during the Stop-signal task. Clin Neurophysiol 120:1717-1723.

Cepeda NJ, Munakata Y (2007) Why do children perseverate when they seem to know better: graded working memory, or directed inhibition? Psychon Bull Rev 14:1058-1065.

Chambers CD, Garavan H, Bellgrove MA (2009) Insights into the neural basis of response inhibition from cognitive and clinical neuroscience. Neurosci Biobehav Rev 33:631-646.

Claffey MP, Sheldon S, Stinear CM, Verbruggen F, Aron AR (2010) Having a goal to stop action is associated with advance control of specific motor representations. Neuropsychologia 48:541-548.

Coxon JP, Stinear CM, Byblow WD (2006) Intracortical inhibition during volitional inhibition of prepared action. J Neurophysiol 95:3371-3383.

Coxon JP, Stinear CM, Byblow WD (2007) Selective inhibition of movement. J Neurophysiol 97:2480-2489.

Curtis CE, D'Esposito M (2008) The inhibition of unwanted actions. In: The psychology of action (Bargh J, Gollwitzer P, Moresella E, eds). New York: Guildford.

Davranche K, Tandonnet C, Burle B, Meynier C, Vidal F, Hasbroucq T (2007) The dual nature of time preparation: neural activation and suppression revealed by transcranial magnetic stimulation of the motor cortex. Eur J Neurosci 25:3766-3774.

D’Esposito M (2007) From cognitive to neural models of working memory. Philos Trans R Soc Lond B Biol Sci 362:761-772.

Diamond A (1990) Developmental time course in human infants and infant monkeys, and the neural bases of inhibitory control in reaching. Ann NY Acad Sci 608:637-676

Duque J, Lew D, Mazzocchio R, Olivier E, Ivry RB (2010) Evidence for two concurrent inhibitory mechanisms during response preparation. J Neurosci 30:3793-3802.

Hoshiyama M, Kakigi R, Koyama S, Takeshima Y, Watanabe S, Shimojo M (1997) Temporal changes of pyramidal tract activities after decision of movement: a study using transcranial magnetic stimulation of the motor cortex in humans. Electroencephalogr Clin Neurophysiol 105:255-261.

MacLeod C, Dodd M, Sheard E, Wilson D, Bibi U (2003) In opposition to inhibition. In: The psychology of learning and motivation, Vol 43 (Ross BH, ed), pp 163-214. San Diego: Elsevier.

Mink JW (1996) The basal ganglia: focused selection and inhibition of competing motor programs. Prog Neurobiol 50:381-425.

Müller NG, Knight RT (2006) The functional neuroanatomy of working memory: contributions of human brain lesion studies. Neuroscience 139:51-58.

Nieuwenhuis S, Yeung N (2005) Neural mechanisms of attention and control: losing our inhibitions? Nat Neurosci 8:1631-1633.

Prut Y, Fetz EE (1999) Primate spinal interneurons show pre-movement instructed delay activity. Nature 401:590-594.

Smith R (1992) Inhibition: history and meaning in the sciences of mind and brain. Berkeley: University of California.

Sohn YH, Wiltz K, Hallett M (2002) Effect of volitional inhibition on cortical inhibitory mechanisms. J Neurophysiol 88:333-338.

Verbruggen F, Logan GD (2009) Models of response inhibition in the stopsignal and stop-change paradigms. Neurosci Biobehav Rev 33:647-661. 